\title{
Traditional protection ratios in FM sound broadcasting - still appropriate for interference management?
}

\author{
J. Philipp \\ Südwestrundfunk Stuttgart, Germany
}

\begin{abstract}
A detailed analysis of the measurement procedures recommended by the International Telecommunication Union (ITU) shows that - with proper definition of audio quality - the FM broadcasting system can provide an audio signal-to-noise ratio of no better than $40 \mathrm{~dB}$, when the interference in the neighboring channels exhausts the limits established by the internationally agreed protection ratios. Thus any attempt to relax the protection, be it motivated by the desire to implement additional FM or new digital services in the FM band, would inevitably degrade reception quality of existing services to levels hardly acceptable by broadcast listeners.
\end{abstract}

\section{Introduction}

The situation of FM broadcasting in Europe is characterized by vast congestion of the allocated spectrum (band II, 87.5 MHz-108.0 MHz). Any attempt to implement new FM stations or digital services in the band almost inevitably is condemned to fail, since the existing services can legally claim protection from any additional interference. The extent to which interference from other FM signals has to be tolerated is governed by mandatory protection ratios, which have been agreed by the ITU conference held in 1984 in Geneva (ITU, 1984). The protection ratios specify the minimum difference of field strength level between wanted and interfering signal, depending on carrier frequency difference. Basically the protection ratio is a receiver property; depending on the technical characteristics of a specific receiver model specific protection ratios have to be complied with to ensure a satisfying quality of the received audio signal. The ITU protection ratios refer to a certain reference audio quality and the properties of some kind of "typical" or "average" receiver, which have been derived from the home receiver population of the 1970s.

In view of the large number of program providers which under the current interference management regime cannot be allocated a band II frequency, it is by no means surprising that periodically the protection ratios are questioned. Two arguments are put forward to support a substantial relaxation. On the one hand, it is said, modern receivers come with considerably improved selectivity compared to 1970 s receivers, and on the other hand, the weighted audio signal-to-noise ratio (SNR) of $50 \mathrm{~dB}$ used to define the ITU protection ratios is not realizable anyway in present-day broadcasting practice (Steil, 2008).

Concerning the first argument it has to be admitted that there are indeed modern receiver models which rival or outperform the 1970s flagships. The majority of receivers actually in use in the average subscriber household consists, however, of low cost equipment the quality of which is not better than or even falls far short of the average 1970 domestic receiver. Car radios constitute a special case. They do have enhanced selectivity (Kammeyer, 1992) and might thus prove that ITU protection ratios are too restrictive. However, the narrow IF bandwidth is associated with increased distortion, which, in the noisy environment of the car, is less audible than increased interference. With car radios as reference for the definition of protection ratios, domestic reception would be impaired by either unacceptable interference or unacceptable distortion, both choices not really being adoptable.

The second argument seems intriguing at first glance. A careful analysis of the measurement process used to define protection ratios in combination with a practical definition of FM broadcasting audio quality reveals, however, that even with protection ratios in force the performance of the FM system is such that any further degradation would hardly be accepted by the subscriber. 


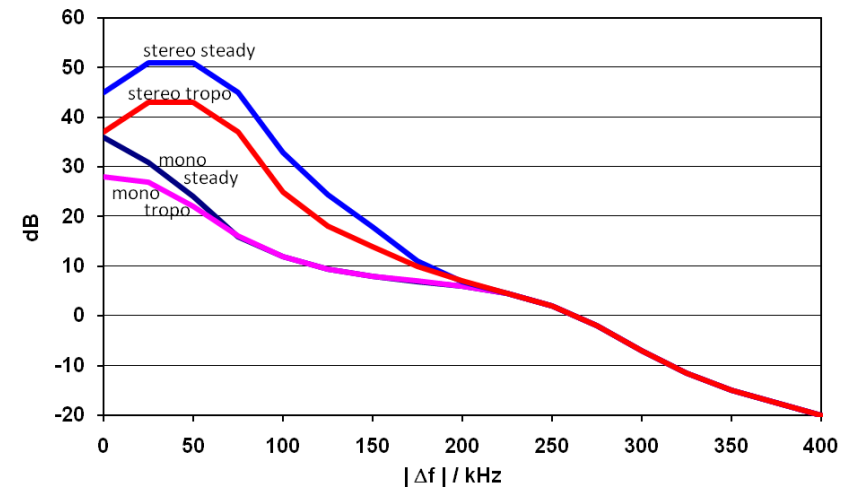

Fig. 1. Protection ratios according to Recommendation ITU-R BS 412-9 as functions of carrier frequency difference. The values for steady interference preserve an undisturbed audio quality, while the values for tropospheric interference, which occurs during small fractions of time only, refer to a level of disturbance which is perceptible, but not annoying.

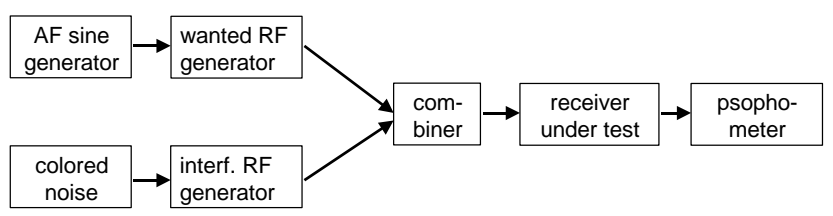

Fig. 2. Basic setup for measurement of the protection ratios applicable to a specific receiver. Reference levels of the wanted signal are established by a $500 \mathrm{~Hz}$ sine test signal. The interferer carries colored noise modulation which simulates a real life program signal. Noise voltage is measured under the condition of modulation pauses, with the wanted modulation switched off.

\section{Protection ratios FM/FM - overview}

The protection ratios laid down in the Geneva 1984 Final Acts reflect values originally published in a recommendation of the CCIR. The current version of this text is Recommendation ITU-R BS 412-9 (ITU, 1998). Figure 1 displays the ITU protection ratios for different carrier frequency offsets between wanted and interfering FM signals. Apparently the stereo signal requires considerably more protection compared with a mono signal. Two types of protection ratio curves are shown for each mode of operation, one for steady and one for tropospheric interference, with slightly relaxed values for the latter case. The reason for the existence of two curves is the fact that in the case of interference, which is harmful only during a small fraction of time, a slight degradation in audio quality can be tolerated. The decision whether an interfering signal has to be treated as steady or tropospheric is taken using the concept of "nuisance field". The nuisance field, defined as sum of interfering field level and protection ratio, is the minimum wanted signal level which provides coverage. If the nuisance field composed of "steady" protection ratio plus time median in-

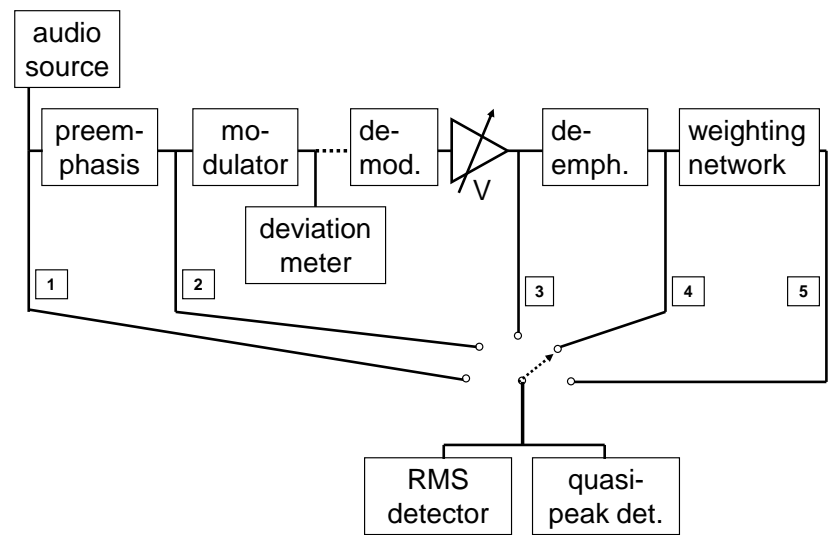

Fig. 3. Signal flow used in CCIR 641 measurement procedure. The (virtual) amplifier $\mathrm{V}$ normalizes the product of modulator and demodulator constants to unity, thus enabling the signal levels at all 5 test points be referred to the same absolute level $0 \mathrm{dBr}$ (see text). The psophometer is shown decomposed into its 3 building blocks weighting network, RMS detector, and quasi-peak detector.

terference level exceeds the nuisance field composed of "tropospheric" protection ratio plus the level of the interfering field which is exceeded in $1 \%$ of time, the interference is classified as steady. While protection from steady interferers aims at providing an audio signal without noticeable interference, the "tropospheric" protection allows disturbances which are perceptible, but not annoying.

The reason given for the vanishing difference between "steady" and "tropospheric" protection ratios for larger carrier frequency offsets is the observation that in these cases a slight increase in interference level causes a disproportionate degradation of audio quality. If this holds true remains to be clarified.

\section{Protection ratios FM/FM - definition and measurement}

According to Recommendation 412 the protection ratios for steady interference provide $50 \mathrm{~dB}$ audio signal-to-noise-ratio (weighted quasi-peak value), measured according to Recommendation ITU-R BS.468-4 (ITU, 1986) with a reference signal at maximum deviation. This is to be understood as the primary definition of protection ratios. It implies a certain value of the practical audio SNR, which will be evaluated in the following. This will be done by careful analysis of the measurement procedure, which has been developed by the Institut für Rundfunktechnik in Munich (Mielke, 1975) as refinement of earlier attempts to relate perceived audio quality with radio frequency S/I (Gramatke, 1958). The measurement procedure finally has attained an official status by publication as Recommendation 661 of the CCIR (CCIR, 1986), which we will adhere to for our analysis. Since the relevant conclusions can already be drawn from the analysis 
of monophonic reception, the discussion will not explicitly deal with the stereo case.

Figure 2 shows the basic setup. It consists of two RF generators, one each for the wanted and the interfering signal. The former can be frequency modulated by an AF sine generator, while the modulation of the latter consists of a colored noise signal defined in Recommendation ITU-R BS.559-2 (ITU, 1990). Both RF signals are combined and fed into the receiver under test. The demodulated $\mathrm{AF}$ signal is measured by a psophometer as specified in Recommendation ITU-R BS.468-4 (ITU, 1986), equipped to display weighted and unweighted rms and quasi-peak audio levels. The filter characteristic of the psophometric weighting network corresponds to the frequency response of the human ear. In the absence of modern methods of psychoacoustical modeling this seems to be the consequent and logical way to objectify subjective perception. Quasi-peak metering is applied to account for the fact that the annoyance of added disturbance is dominated by its peaks rather than its rms value.

For the detailed assessment of the various signal levels relevant in the measurement of the protection ratios we refer to Fig. 3, which shows the signal path across the relevant functional blocks. The entire RF part of the transmission chain between modulator and demodulator can - despite of the fact that especially the front end and IF amplifier determine the receivers' susceptibility to interference - be ignored as far as the measurements are concerned. It has to be stressed that in the measurement process relative signal levels are of relevance only, in particular differences constrained to the side of the transmitter and the receiver, respectively. Any complication by criss cross differences will not be inferred. For the analysis therefore we can without loss of generality take the assumption that the combined effect of modulator and demodulator constants is cancelled by a normalizing amplifier which is adjusted as to make signal levels of modulator input and demodulator output equal. This adjustment results, of course, in equal levels of the audio signal before pre- and after deemphasis. After all, all relevant audio signal levels can be measured on a common scale the zero of which is chosen as $0 \mathrm{dBr}$. This corresponds, according to the specifications of Recommendation ITU-R BS.412-9 (ITU, 1998) to the power of a sinusoidal signal which, when fed into the modulator, gives rise to a peak frequency deviation of $\pm 19 \mathrm{kHz}$.

The actual protection ratio measurement is done in 4 steps:

1. Adjusting the level of the reference AF signal to obtain a defined peak frequency deviation

2. Adjusting the RF level of the wanted transmitter and noting the level of the received reference AF signal (interfering transmitter off)

3. Adjusting the level of the noise signal to obtain a defined frequency deviation

4. Adjusting the RF level of the modulated interfering signal (at carrier frequency offset under investigation,

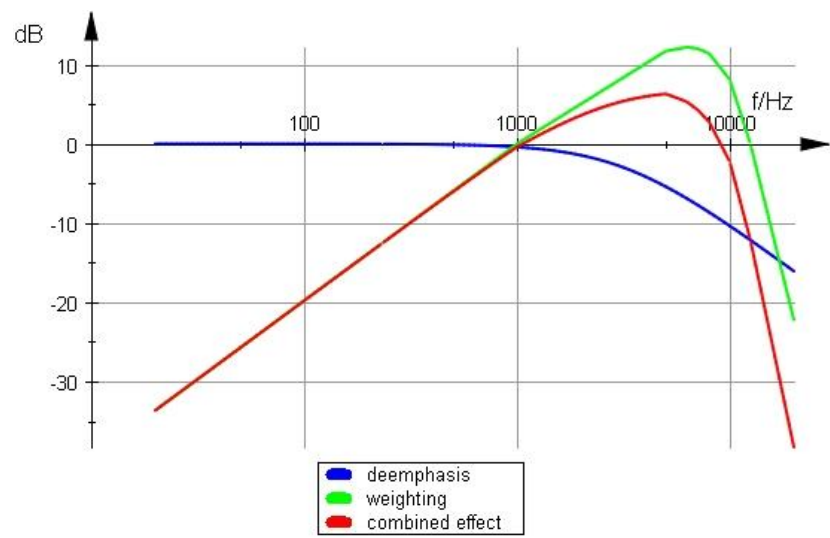

Fig. 4. Frequency response of deemphasis filter, psophometer weighting network, and combined effect.

modulation of the wanted transmitter switched off) until the weighted quasi-peak level of the resulting AF disturbance is $50 \mathrm{~dB}$ below the reference level established in step 2

In step 1 the RF generator which is disposed for the delivery of the wanted signal is frequency modulated by a sinusoidal signal of $500 \mathrm{~Hz}$. The amplitude of this signal is adjusted to produce a peak frequency deviation of $\pm 75 \mathrm{kHz}$. Thus we note that the unweighted rms level of the audio signal at the modulator input (test point 2 in Fig. 3) is given by

p_ref ${ }_{2}=20 \mathrm{dBr} \lg (75 / 19)=11,9 \mathrm{dBr}$

To obtain the corresponding level of the audio signal before preemphasis (test point 1) we have to subtract the boost caused by the preemphasis (we will make use of preemphasis with a time constant of $50 \mu \mathrm{s}$ ) which for $500 \mathrm{~Hz}$ amounts to just $0.1 \mathrm{~dB}$ (cf. deemphasis characteristic shown in Fig. 4):

p_ref $_{1}=$ p_ref $_{1}-0.1 \mathrm{~dB}=11.8 \mathrm{dBr}$

In step 2 the level of the wanted RF signal is adjusted sufficiently high to provide an audio SNR of at least $56 \mathrm{~dB}$ and sufficiently low to avoid complications by receiver front end nonlinearity. Due to our normalization, the unweighted level of the received reference AF signal (test point 4) equals the level at test point 1 :

p_ref $_{4}=$ p_ref $_{1}=11.8 \mathrm{dBr}$

In step 3 the level of the noise modulation of the interfering transmitter is adjusted to mimic a realistic interfering signal. This turns out to be not completely straightforward since, at the outset, noise is not a truly realistic modulating signal in broadcasting. However, real program signals will render the psophometer needle wiggling and not facilitate reliable readings (we tried with "All the Things You Are" as played by Dave Brubeck and found quasi-peak readings fluctuating by $\pm 5 \mathrm{~dB}$ ). Therefore, colored noise with the same spectrum 


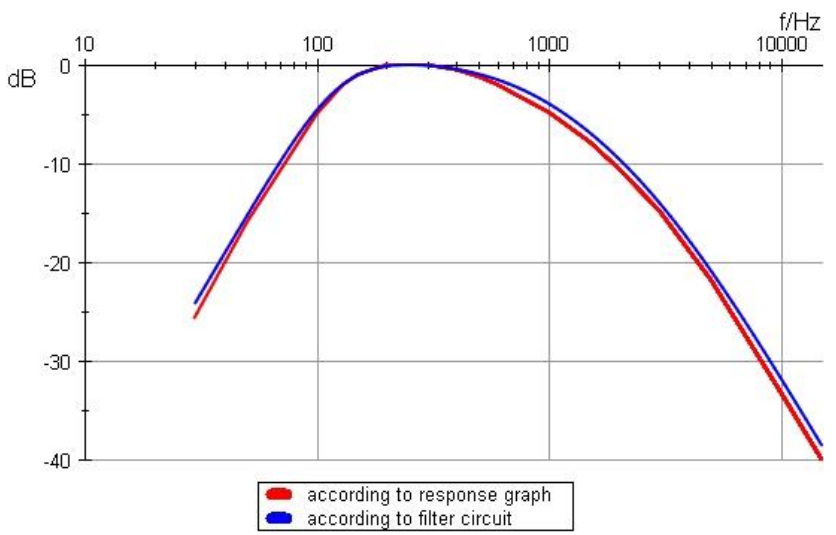

Fig. 5. Colored noise spectrum defined in Recommendation ITUR BS.559-2. The two definitions (frequency response of the filter used to transform white noise into standard colored noise, copied from the presented graph and computed from the component values of the explicit filter circuit) differ by less than $1 \mathrm{~dB}$.

as a typical uncompressed program signal ("modern" dance music being the prototype) has been specified as a replacement. Its spectral shape is defined in Recommendation ITUR BS.559-2 (ITU, 1990) both in the form of a specification of the resistances and reactances of a filter circuit which converts white to standard colored noise, and in the form of a graphical representation of the frequency response of the filter. The latter and the response calculated from the filter component values do not completely coincide, but the difference generally is below $1 \mathrm{~dB}$ (Fig. 5).

To adjust the noise level, first a $500 \mathrm{~Hz}$ sine signal is used to modulate the interfering transmitter. Its level is adjusted so as to give rise to a frequency deviation of $\pm 32 \mathrm{kHz}$. Then the colored noise level is adjusted so that its unweighted quasipeak value equals the rms level of the $500 \mathrm{~Hz}$ sine signal (the quasi-peak psophometer reading is defined as equivalent rms level; for sine signals, quasi-peak and rms readings are the same). The adjustment thus results in a noise modulation with a "quasi-peak" frequency deviation of approximately $\pm 32 \mathrm{kHz}$ (slightly more due to preemphasis).

To determine the actual signal levels involved, we start with the $500 \mathrm{~Hz}$ sine signal. Its level after preemphasis (test point 2) is given by

$p \_n n_{2}=20 \mathrm{dBrlg}(32 / 19)=4,5 \mathrm{dBr}$

The rms level before preemphasis then is - corresponding to Eq. (2), the case of the reference wanted modulation - given by

$p \_n_{1}=p \_n_{2}-0.1 \mathrm{~dB}=4.4 \mathrm{dBr}$

The signal being a sinusoid, its quasi-peak level equals its rms level. Since the noise signal is adjusted to have the same quasi-peak level $p \_n_{1, \mathrm{qp}}$, we have

$p \_n 1, \mathrm{qp}=4.4 \mathrm{dBr}$
Now we have to address the rms level of the noise signal. Unfortunately the specification of the dynamic characteristics of the quasi-peak meter does not easily lend itself to mathematical modeling. Therefore it has been decided to determine the conversion factor in question experimentally. First, two tracks of standard Gaussian colored noise $\left(2^{17}\right.$ samples each, sampling frequency $100 \mathrm{kHz}$, signal duration $1.31 \mathrm{~s}$, channels at multiples of $0.736 \mathrm{~Hz}$ approx.) have been synthesized by inverse Fourier transform of its spectrum with random phases and amplitudes read from the graphical filter response (Fig. 5) of ITU (1990). These signals, made continuous by cyclic repetition, have been fed into three different psophometers (2 Siemens U2033 and 1 Rohde \& Schwarz UPGR) while noting the corresponding quasi-peak and rms readings. It turned out that in the quasi-peak mode the psophometer needle was constantly rocking by slightly more than $\pm 1 \mathrm{~dB}$ when monitoring one track and by $\pm 0.4 \mathrm{~dB}$ when monitoring the other, which rendered unambiguous readings difficult. However, human investigators not surprisingly tend to intuitively average the needle movement. So it was decided to use the "intuitive average" difference between quasipeak and rms in the sequel. It amounted to $4.1 \mathrm{~dB}$ with an estimated uncertainty of $\pm 0.2 \mathrm{~dB}$, with close match between the three psophometers. Thus we can state that for our standard colored noise signal the rms level before preemphasis is given by

$\left.p\lrcorner n_{1}=p\right\lrcorner n_{1, \mathrm{qp}}-4.1 \mathrm{~dB}=0.3 \mathrm{dBr}$

It should be noted that the observed quasi-peak-to-rms ratio can be interpreted in terms of instantaneous amplitude probability. Let the difference between quasi-peak and rms level be designated by $\mathrm{d}$ and let the standard deviation of the instantaneous amplitudes be $\sigma$ and let the amplitude which corresponds to the quasi-peak-level be $a_{\mathrm{qp}}$. The psophometer indication refers to $a_{\mathrm{qp}}^{*}:=a_{\mathrm{qp}} / \sqrt{ } 2$. Then we have

$d=20 \mathrm{~dB} \lg \left(a_{\mathrm{qp}}^{*} / \sigma\right)$

from which we calculate

$a_{\mathrm{qp}}^{*} / \sigma=10^{d / 20 \mathrm{~dB}}$

Since the noise samples are sums of random numbers, their distribution is Gaussian. Thus the probability with which the instantaneous amplitude $x$ exceeds $\pm a_{\mathrm{qp}}$ is given by

$p\left(|x|>a_{\mathrm{qp}}\right)=\operatorname{erfc}\left[a_{\mathrm{qp}} /(\sigma \sqrt{ } 2)\right]=\operatorname{erfc}\left(a_{\mathrm{qp}}^{*} / \sigma\right)$

With $d=4.1 \mathrm{~dB}$ we find that for standard colored noise the quasi-peak amplitude is the value which is exceeded in $2.3 \%$ of time.

To find out the noise signal level at modulator input we have to add the level shift introduced by the preemphasis filter. This has been computed numerically, making use of the synthesized noise signal. A level increase of $0.9 \mathrm{~dB}$ has been found. Thus the noise level at test point 2 is given by

$p\lrcorner n_{2}=p \_n_{1}+0.9 \mathrm{~dB}=1.2 \mathrm{dBr}$ 
This is not a legal modulation signal, since both international (ITU, 1990) and national (e.g., BNetzA, 2009) directives restrict multiplex power to $0 \mathrm{dBr}$. Hence it might be argued that the disturbance introduced by the test noise could be overestimated in the measurements according to CCIR Recommendation 641 (CCIR, 1986). However, assuming that it is peak rather than rms deviation which is responsible for the perceived degree of disturbance, the frequency of occurrence of peaks in the noise modulation should be comparable to the (worst case) frequency of occurrence in real program signals. The latter can be inferred, e.g., from the limit established by the German Federal Network Agency (BnetzA, 2009) which restricts instantaneous deviation of more than $\pm 75 \mathrm{kHz}$ to a fraction of time less than or equal to $1 \times 10^{-6}$. The noise signal, on the other hand, has an rms deviation of $19 \mathrm{kHz} / \sqrt{ } 2 \times 10^{1.2 / 20}=15.4 \mathrm{kHz}$. Thus the probability with which $\pm 75 \mathrm{kHz}$ is exceeded can be computed as $\operatorname{erfc}(75 / 15.4 / \sqrt{ } 2)=1.1 \times 10^{-6}$. This comes close to the legal limit, especially when taking into account the uncertainty in the "intuitive" meter-reading that has been necessary (less than $0.1 \mathrm{~dB}$ correction of noise level would render the match perfect). Thus it can be concluded that the apparent overmodulation is justified.

Admittedly the line of arguments is not strictly valid for the case of co-channel interference into mono transmissions. In this case the disturbance can be largest when the interfering carrier is barely modulated, as can be seen from measurements reported in Mielke (1978) and be derived from the explicit formulas for the demodulated audio signal provided by Kammeyer (Kammeyer, 1992). This is illustrated by Fig. 6, which displays that in the co-channel case (weighted) audio SNR is trending to increase with increasing deviation of the (sine modulated) interfering signal. For the mono co-channel situation, the CCIR 641 procedure might miss to reflect the worst case.

Having established the appropriateness of the noise signal, we now can compute the resulting weighted audio SNR after the demodulator. Within the context of the measurements, audio SNR will be defined as the ratio of the (weighted) rms level of the undisturbed audio signal to the (weighted) quasipeak disturbance during modulation pauses of the wanted transmitter (where auditory masking by the wanted signal does not occur). We first look at the reference modulation $(500 \mathrm{~Hz})$.

From Eq. (3) we recall the level at the input of the weighting network. The network will attenuate the $500 \mathrm{~Hz}$ sine signal by $6.1 \mathrm{~dB}$. Thus the weighted rms audio level of the undisturbed wanted signal (test point 5 ) is given by

p_ref $_{5}=$ p_ref $_{4}-6.1 \mathrm{~dB}=11.8 \mathrm{dBr}-6.1 \mathrm{~dB}=5.7 \mathrm{dBr}$

If we now reduce the test sine modulation to the legal limit of $0 \mathrm{dBr}$, we have to reduce the level by $11.9 \mathrm{~dB}$, according to Eq. (1). Thus with Eq. (12) the weighted audio level of the legal sine signal at test point 5 amounts to

p_leg $500_{5}=$ p_ref $_{5}-11.9 \mathrm{~dB}=-6.2 \mathrm{dBr}$

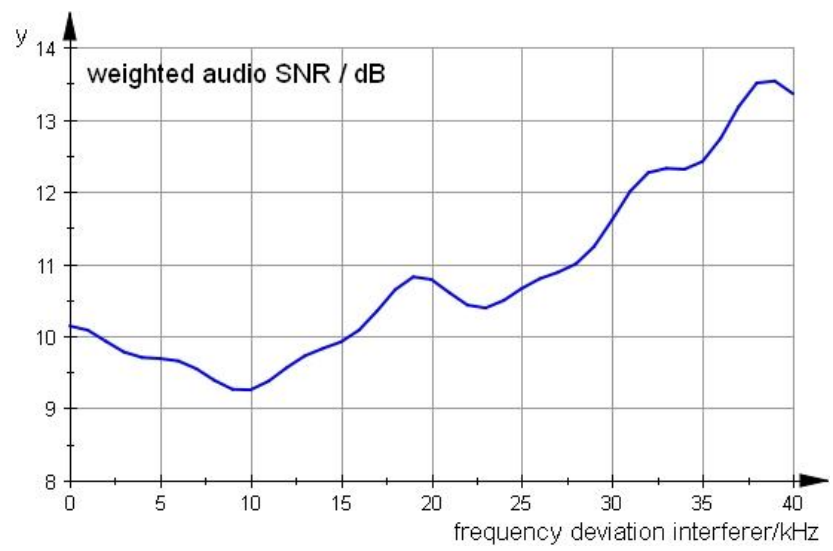

Fig. 6. Weighted audio SNR for co-channel interference. Carrier level ratio: $20 \mathrm{~dB}$, wanted modulation: $500 \mathrm{~Hz}$ sine at $19 \mathrm{kHz}$ max. deviation, interfering modulation: $1980 \mathrm{~Hz}$ sine. Audio SNR is trending to improve with increasing deviation of the interfering signal.

According to the measurement procedure, the weighted quasi-peak disturbance is just $50 \mathrm{~dB}$ below the unweighted reference (3),

$p\lrcorner n_{5, \mathrm{qp}}=\mathrm{p} \mathrm{ref}_{4}-50 \mathrm{~dB}=-38.2 \mathrm{dBr}$

which results in a SNR of a mere p_leg500 $5-p \_n_{5, \mathrm{qp}}=$ $32.0 \mathrm{~dB}$. However, this low value does not directly reflect the apparent quality of the FM system. The reason is the rather artificial choice of the $500 \mathrm{~Hz}$ reference modulation, which can hardly represent real program material, especially with regard to the effect of the weighting network. A better choice would be to use standard colored noise instead.

Let us assume that we modulate the wanted transmitter with standard colored noise using the legal level of $0 \mathrm{dBr}$ at test point 2 . The level boost by preemphasis is, as in Eq. (11), $0.9 \mathrm{~dB}$. Thus we have to set the noise generator to an output of $-0.9 \mathrm{dBr}$. This level will be found again at test point 4 . The effect of the weighting network on the colored noise is a boost of $2.2 \mathrm{~dB}$ (numerical computation). Thus we end up with a weighted rms audio level of the legal realistic wanted signal of $1.3 \mathrm{dBr}$. Compared with the weighted quasi-peak disturbance of $-38.2 \mathrm{dBr}$ (Eq. 14) we finally end up with an SNR of $1.3 \mathrm{dBr}-(-38.2 \mathrm{dBr})=39.5 \mathrm{~dB}$, which is in perfect agreement with the common technicians' notion of FM broadcasting providing a dynamical range of no better than $40 \mathrm{~dB}$.

\section{Conclusion}

The protection ratios developed for FM broadcasting about half a century ago allow for an audio signal-to-noise ratio of approximately $40 \mathrm{~dB}$. Compared to the SNR of $80 \mathrm{~dB}$ and more present-day listeners are accustomed to from modern sound recording media, the FM value seems archaic and 
should definitively not be further deteriorated by any relaxation of the protection ratios.

\section{References}

BNetzA (Bundesnetzagentur, German Federal Network Agency): BnetzA Entwurf 511 MV 07, Messvorschrift (MV) zur Messung von Frequenzhub und Multiplexleistung von UKW-TonRundfunksendern an der Luftschnittstelle, Amtsblatt der Bundesnetzagentur, Bonn, 3029-3046, 2009.

CCIR (Comité Consultatif International des Radiocommunications): Recommendation 641, Determination of radio-frequency protection ratios for frequency-modulated sound broadcasting, Geneva (also available as ITU-R Rec. BS.641), 1986.

Gramatke, B., Netzband, R., und Paulsen, E.: Erforderliche HFStörabstände bei modernen UKW-FM-Empfängern, RTM 2, 4153, 1958.
ITU (International Telecommunication Union): Final acts of the Regional Administrative Conference for the Planning of VHF Sound Broadcasting (Region 1 and Part of Region 3), Geneva, 1984.

ITU (International Telecommunication Union): Recommendation ITU-R BS.468-4, Measurement of audio-frequency noise voltage level in sound broadcasting, Geneva, 1986.

ITU (International Telecommunication Union): Recommendation ITU-R BS.559-2, Objective measurement of radio-frequency protection ratios in LF, MF and HF broadcasting, Geneva, 1990.

ITU (International Telecommunication Union): Recommendation ITU-R BS.412-9, Planning standards for terrestrial FM sound broadcasting at VHF, Geneva, 1998.

Kammeyer, K. D.: Nachrichtenübertragung, Stuttgart, 1992.

Mielke, E.-J.: Ein objektives Zweisignal-Messverfahren zur Messung von RF-Störabständen an FM-Tonrundfunkempfängern, RTM 19, 110-119, 1975.

Steil, A., Schad, F., and Lehnert, J.: Neue Wege zur Digitalisierung des Hörfunks mit DRM+, FKT 62, 645-650, 2008. 